\title{
Mesh Based Multicast Routing in MANET: Stable Link Based Approach
}

\author{
Rajashekhar Biradar, Sunilkumar Manvi, Member, IACSIT, Mylara Reddy
}

\begin{abstract}
The group-oriented services are one of the primary application classes that are addressed by mobile ad hoc networks (MANETs) in recent years. To support such services, multicast routing is used. Thus, there is a need to design stable and reliable multicast routing protocols for MANETs to ensure better packet delivery ratio, lower delays and reduced control overheads. In this paper, we propose a mesh based multicast routing scheme that finds stable multicast path from source to receivers. The multicast mesh is constructed by using route request and route reply packets with the help of multicast routing information cache and link stability database maintained at every node. The stable paths are found based on selection of stable forwarding nodes that have high stability of link connectivity. The link stability is computed by using the parameters such as received power, distance between neighboring nodes and the link quality assessed using bit errors in a packet. The proposed scheme is simulated over a large number of MANET nodes with wide range of mobility and the performance is evaluated. It is observed that proposed scheme produces better packet delivery ratio, less control overheads and reduced packet delay compared to on-demand multicast routing protocol (ODMRP).
\end{abstract}

Index Terms - MANET, multicast routing, stable forwarding node, routing information cache, link stability database.

\section{INTRODUCTION}

The characteristic of mobile ad hoc networks (MANETs) is that they do not have fixed network infrastructure, nodes can act as both host and router, nodes may be mobile, nodes may have limited resources, limited battery life and they have capability of self organization. MANETs require fundamental changes to conventional routing protocols for both unicast and multicast communication owing to its unique features. With the rapid growth of group communication services, the multicast routing in MANET has attracted a lot of attention recently [1][2][3][4][5]. In multicast routing, a path is set up connecting all group members so that bandwidth is not wasted. Group communication applications include audio/video conferencing as well as one-to-many data dissemination in critical situations such as disaster recovery or battlefield scenarios. Also, their applications are felt in mobile/wireless environments where the mobility and topology changes produces very high overhead and affects the throughput

Manuscript received July 27, 2009.

Rajashekhar Biradar and Sunilkumar Manvi are with the Department of Electronics and Communication Engineering and Mylara Reddy is with the Department of Information Science and Engineering. All three authors are with Reva Institute of Technology and Management, Bangalore-560064, India. (Phone number: +91-80-65687563)

e-amil: \{raj.biradar, sunil.manvi, mylarareddy\}revainstitution.org performance in terms of packet delivery ratio. Since group-oriented communication is one of the key application classes in MANET environments, a number of MANET multicast routing protocols have been proposed. These protocols are classified according to two different criteria. The first criterion maintains routing state and classifies routing mechanisms into two types: proactive and reactive. Proactive protocols maintain routing state, while the reactive protocols reduce the impact of frequent topology changes by acquiring routes on demand. The second criterion classifies protocols according to the global data structure that is used to forward multicast packets. Existing protocols are either treeor mesh-based. Tree-based schemes establish a single path between any two nodes in the multicast group. These schemes require minimum number of copies per packet to be sent along the branches of the tree. Hence, they are bandwidth efficient. However, as mobility increases, link failures trigger the reconfiguration of the entire tree. When there are many sources, one either has to maintain a shared tree, losing path optimality, or maintain multiple trees resulting in storage and control overhead. Examples of tree-based schemes include [6][7][8]: ad hoc multicast routing protocol (AMRoute), ad hoc multicast routing utilizing increasing ID-numbers protocol (AMRIS), and multicast ad hoc on-demand distance vector routing protocol (MAODV).

Mesh-based schemes establish a mesh of paths that connect the sources and destinations. They are more resilient to link failures as well as to mobility. The major disadvantage is that

mesh-based schemes introduce higher redundancy of packets since multiple copies of the same packet are disseminated through the mesh, resulting in reduced packet delivery and increase in control overhead under highly mobile conditions. Some examples of mesh-based schemes are (a) on demand multicast routing protocol (ODMRP [9]), (b) forwarding group multicast protocol (FGMP[10]), (c) core assisted mesh protocol (CAMP[11]), ((d) neighbor supporting ad hoc multicast routing protocol (NSMP[12]), (e) location-based multicast protocol[13], and (f) dynamic core-based multicast protocol (DCMP[14]). In ODMRP, a source periodically floods an advertising packet in the network. A receiver responds to this packet by using backward learning. The nodes on the path from the receiver to the source form a mesh of forwarding nodes for the multicast group and thus ODMRP is one of the well established protocols. The major advantage of ODMRP is that it produces high packet delivery ratio and throughput even under highly mobile network conditions. The disadvantage of ODMRP is that the control overhead also grows higher and higher with network size [15][16][17][18].

The work given in [15] solves the problem of limiting control and data overhead for mesh based multicast routing. It 
defines the mean link duration metric to adapt and reduce refreshing control packets and also suggests a new reactive multicast mesh construction algorithm with overhearing technique that forms a fish bone structure. Each mesh member chooses its forwarding node independently and entirely in a distributed fashion, based on its own perceived network conditions to provide a tradeoff between reducing data overhead and achieving multicast reliability. The work given in [4] proposes query packets containing source id, sequence number, next sequence number, hop count and the time interval needed to send next query packet. Query packet is sent by multiple sources and is processed by intermediate nodes and receivers.

In [17], an on demand multicast routing protocol named as source routing based multicast protocol (SRMP) is presented. This protocol constructs a mesh of paths to connect group members, providing robustness against mobility. It also provides stable paths based on link availability according to future prediction of links state, and higher battery life paths tending to power conserving. SRMP does not use periodic network flooding of control packets but instead, it constructs a stable mesh based upon an estimate of future link availability and thus enhances battery life and minimizes the possibility of link failures and finally reduces the overhead needed to reconstruct the paths compared to ODMRP and ADMR protocols.

Reference [19] proposes a cluster-based on demand multicast routing protocol (SC-ODMRP) as an extension to the flat multicast routing protocols in large scale ad hoc networks using clustering concept on ODMRP to improve network performance in terms of end-to-end delay and control packets. The paper also proposes a link stability approach to design a stable multicast algorithm. This approach increases data delivery and decreases overhead.

Reference [20] proposes a cluster based stable multicast routing protocol (CBSRP) in Ad Hoc Networks. The protocol uses flooding algorithm under extended range of some network conditions like higher mobility and enhanced traffic conditions. It constructs a new metric of node stability and selects a stable path with the help of entropy metric to reduce the number of route reconstruction. It selects the nodes which have the most weight and stability to be the cluster heads. Though the stable node selection increases the stability but this selection is made possible using clusters and the proactive maintenance of cluster heads is a major overhead and the overhead increases with number of nodes.

In our previous work [21], a mesh based multicast routing scheme is discussed which establishes a multicast mesh on demand. The work is not supported with validation of the scheme and performance analysis and also lacked proper formulation of components of the scheme. This paper provides an extension to the work by providing detailed functioning of the scheme, examples and simulation based performance analysis.

As per the literature survey, it is observed that multicast routing protocols try to achieve better performance in terms of packet delivery ratio, reliability, less control overheads and packet delays. The performance is better in mesh based protocols as they provide redundant routes and less prone to packet loss under mobility. Since ODMRP is one of the well studied protocols, it inherits all the merits of mesh based protocols. However, performance of ODMRP can be further improved by considering stable links during mobility conditions that reduces packet loss in case of link or node failures. Without stable links, the paths established are vulnerable due to large mobility patterns of nodes. Thus, there is a need to develop an efficient link stability based multicast routing scheme that provides better packet delivery ratio, delays and control overheads. In this paper, we design a link stability based multicast routing scheme that uses stable links by selecting stable forwarding nodes (SFNs), and the simulation results are compared with that of ODMRP.

\section{A. Proposed Work}

In this paper, we propose a link stability based multicast routing scheme that establishes a route from source to multicast destinations in MANET. A multicast mesh is created with stable links when a source node needs to send data to receiver nodes. The scheme consists of following phases.

1) Mesh creation through the route request (RR) packets and route reply (RP) packets, 2) finding stable routes between source to destination by selecting SFNs using link stability metric, 3) mesh maintenance and handling link failures. The link stability is computed using power received at a node, distance between nodes and the packet losses. Our contributions in this paper are as follows. 1) Defining route request and route reply packets to create a mesh by using transmission power and antenna gains. 2) Creation and maintenance of routing information for hop by hop routing for a multicast connection by using route request and route reply packets based on link stability. 3) Selecting stable forwarding

node for multicast paths based on link stability computed using the parameters such as received power, distance between the nodes and link quality. 4) Attempts to select different stable forwarding node in a mesh during link failures rather than immediately going in for route discovery. 5) Comparing the performance of the proposed scheme with ODMRP.

\section{B. Organization of the Paper}

The rest of the paper is organized as follows. Section 2 presents the proposed link stability based multicast routing scheme in MANET in which the details of multicast mesh creation is discussed using route request, route reply packets to establish stable path with multicast routing information cache and link stability database. Section 3 presents simulation environment comprising network model, channel model, mobility model and traffic model along with parameters used for simulation. Section 4 discusses simulation results and comparison with ODMRP. Section 5 concludes the work.

\section{LinK Stability BASED MulticAst Routing}

This section presents the functioning of proposed link stability based multicast routing scheme in MANET (LSMRM). Here, we discuss the process of creating a mesh of multicast routes with the help of RR and RP packets, routing information maintained in multicast routing information cache (MRIC) and link stability database. MRIC 
is maintained at every node. After creating a multicast mesh, a stable route between source destination pair is established by using SFNs (which are a part of multicast mesh) that have stable link connectivity. Link stability database is maintained at every node, which stores the updated information that is used for finding stable multicast routes in a mesh.

\section{A. Route Request, Route Reply and Route Error Packets}

To create a multicast mesh and a stable route in a mesh from source to destination, various control packets such as route request, route reply and route error (RE) packets are used. In this section, we describe some of the fields of the control packets required for multicast mesh creation, stable path establishment and handling link failure situations. The fields of RR packet are as follows.

- Source address: It is the address of the node originating the packet.

- Multicast group address: It is the address of the multicast group.

- Sequence number: The sequence number assigned to every packet delivered by the source that uniquely identify the packet.

- Route request flag (RR flag): This flag is set for the duration of forward travel of RR packet from source to destination.

- Previous node address: It is the address of previous node that RR packet has visited during its forward movement. In the route request phase, a node receiving $\mathrm{RR}$ packet stores this address with multicast address in its MRIC as next hop node to send the packets to RR packet source. This field is updated after every movement to the next node until it reaches the receiver with multicast address.

- Power: This is the power at which a node has transmitted the packet to neighbor.

- Antenna gain: This is gain of antenna at the forwarding node to forward RR packet to its neighbor.

RP packet format for multicast mesh creation is almost similar to RR packet with few changes in RR packet. They are as follows: RR flag value will be made 0 , previous node address is removed, and source address is replaced by receiver address. RP packet moves on path traversed by RR packet by using MRIC and also updates the MRIC towards receiver /multicast address by adding one more next hop (node address from where RP packet has come) to multicast address. In general, next hop at every node to reach a source is set by using RR packets whereas RP packets set next hop at every node to reach receivers from the source. RE packet is generated when a node is unable to send the packets. Some of the fields of this packet are source address, destination address, sequence number, and route error flag (RE flag). Whenever a node identifies link failures, it generates RE packet with route error flag set and sends the packet to either source or receiver. If link failure occurs in forward journey of a RR packet from source to multicast receiver, RE packet is sent to the source and if link failure occurs for reverse journey

of the RP packet from receiver to the source, RE packet is sent

to the multicast receiver.

\section{B. Multicast Routing Information Cache (MRIC)}

Each node in the network maintains its own MRIC that aids in forwarding packets to group members. A node adds information to its MRIC as it learns of new routes for various multicast groups in MANET; for example, a node may update

new routes when it receives $\mathrm{RR}$ and $\mathrm{RP}$ packets, and likewise.

A node removes information from its MRIC as it learns that existing routes in the ad hoc network have failed due to link and node failures. For every visited packet (RR or RP) at a node, MRIC is updated with some of the following fields required for establishing multicast mesh and stable paths (see Figure 1).

\begin{tabular}{|c|c|c|c|c|}
\hline $\begin{array}{c}\text { GROUP } \\
\text { ID/DST. } \\
\text { ADDR. }\end{array}$ & $\begin{array}{c}\text { NEXTHOP } \\
\text { ADDR. }\end{array}$ & $\begin{array}{c}\text { FW } \\
\text { FLAG }\end{array}$ & $\begin{array}{c}\text { STABILIT } \\
\text { Y FACTOR }\end{array}$ & $\begin{array}{c}\text { SEQ. } \\
\text { NO. }\end{array}$ \\
\hline $228.10 .10 .0 /$ & 128.80 .10 .1 & 01 & 0.7 & 100 \\
92.19 .10 .10 & 128.80 .0 .80 & 01 & 0.9 & 105 \\
& 128.80 .0 .11 & 10 & 1.0 & 234 \\
\hline$\ldots$ & $\ldots$ & $\ldots$ & $\ldots$ & $\ldots$ \\
\hline
\end{tabular}

Fig. 1. Multicast routing information cache

- Group address and Destination address: Group address is the address of multicast group. Destination address is the address of the node where packet has to be forwarded with multicast address. This helps to accommodate the routes created by RR packets and RP packets.

- Next hop addresses: These are the addresses of the next hop interfaces for forwarding to a multicast group.

- Forwarding flag (FW flag): This field stores two bit flag that indicates the status of node in three modes; mode 00node is multicast group node, mode 01 - node is a forwarding node and mode 10 - node is a forwarding node and is on the stable path.

- Sequence number: This is the number given by the node which has a route to multicast receivers. It helps in differentiating the time order in which route is created. A node updates routes if the received sequence number in $\mathrm{RR} / \mathrm{RP}$ packet is higher than the existing sequence number. It is set to infinity if a next hop link fails.

- Next hop stability: This defines stability factor of a link connecting next hop (taken from link stability database). FW flag for a forwarding node of a multicast group will be set to 10 if the node has high stability factor compared to other next hops. In figure 1 , stable next hop used for forwarding to multicast address/ destination address 228.10.10.0/92.19.10.10 is 128.80 .0 .110 .

\section{Link Stability Database}

Each node maintains a link database information which helps in establishing and maintaining multicast mesh and stable path from source to multicast destinations. It maintains the following parameters: node ID, power level, distance, and link quality and stability factor.

- Node ID: It stores the neighbor node ID.

- Power level: Whenever a packet (either RR or RP packet) is received from its neighbor, this field stores the ratio 
$\left(P w_{i j}\right)$ of measured value of the power received $\left(P_{r}\right)$ at the node to the power transmitted $\left(P_{t}\right)$ by neighbor node.

- Distance: This field stores the distance between the neighboring nodes. The distance is computed by using the free space propagation model [22][23] given in equation 1 .

$$
P_{r}(d)=\frac{P_{t} G_{t} G_{r} \lambda}{(4 \pi) d^{2} L}
$$

where $G t$ and $G r$ are the antenna gains of the transmitter and the receiver, respectively. $L$ is the system loss, $\lambda$ is the wavelength and $d$ is the distance between two MANET nodes.

- Link quality: This field stores the value of the link quality of neighboring nodes. It is approximated by ratio of the number of bits in error to the number of bits received (bit error rate). This value gets updated for every packet received at a node over a certain period. It depends on parameters such as the interference effect of the wireless channel, additive white Gaussian noise, and signal transmission range.।

- Stability factor: It is the value computed for a link to a neighbor based on the power level, distance and link quality. Stability factor $S_{i j}$ of a link between nodes $i$ and $j$ is defined by equation 2 .

$$
S_{i j}=\left[\frac{P w_{i j} \times q_{i j}}{d_{i j}}\right]
$$

where $P w_{i j}, q_{i j}$ and $d_{i j}$ are the signal strength, link quality and the distance between nodes $i$ and $j$, respectively.

\section{Multicast Mesh Creation}

Multicast mesh creation involves two phases; a request phase and a reply phase. Request phase invokes a route discovery process to find routes to the multicast group. Different routes to the multicast group are setup during the reply phase. There are two types of nodes defined based on whether they are multicast group members or non-group members. Group members include all multicast sources, receivers and that of non-group members include intermediate nodes that help to create multicast routes from source to receivers. Non-group members help in forwarding the data packets. Both group members and non-group members help in recovery of failed links due to mobility of nodes and other interferences in route maintenance phase.

LSMRM identifies some of the intermediate nodes from the forwarding group as SFN's that forms stable multicast path based on link stability. Any non-group member may join the group by registering itself as a group member. Registration is made by sending RR packet to its neighbors and getting the RP packet from any one of the group members or forwarding nodes in its neighborhood. Once a non-group member receives RP packet from neighbor group member node, it becomes a group member. In the following section, we discuss the process of request phase, reply phase that helps in creating multicast mesh.

1) Request Phase: A source finds the multicast routes to its receivers by using $R R$ packets. The sequences of operations that occur are as follows. 1) Source prepares a RR packet by putting the following information: its address, multicast destination address, set $\mathrm{RR}=1$, sequence number, previous node address as its address, transmission power and antenna gain. 2) Broadcast RR packet to neighbors. 3) A node receiving $R R$ packet will discard it if it is already received (by using sequence number and source address). 4) If RR packet is not a duplicate, update the MRIC and link stability database: checks MRIC for availability of route for multicast address / source address, if available and the sequence number of RR packet is higher than the MRIC sequence number then update the next hop for multicast address / source address as previous node address with new sequence number. 5) Rebroadcast the RR packet to its neighbors. 6) Perform steps 3 to 5 until receiver is reached. 7) If receiver is not reached within certain hops, send RE packet to source.

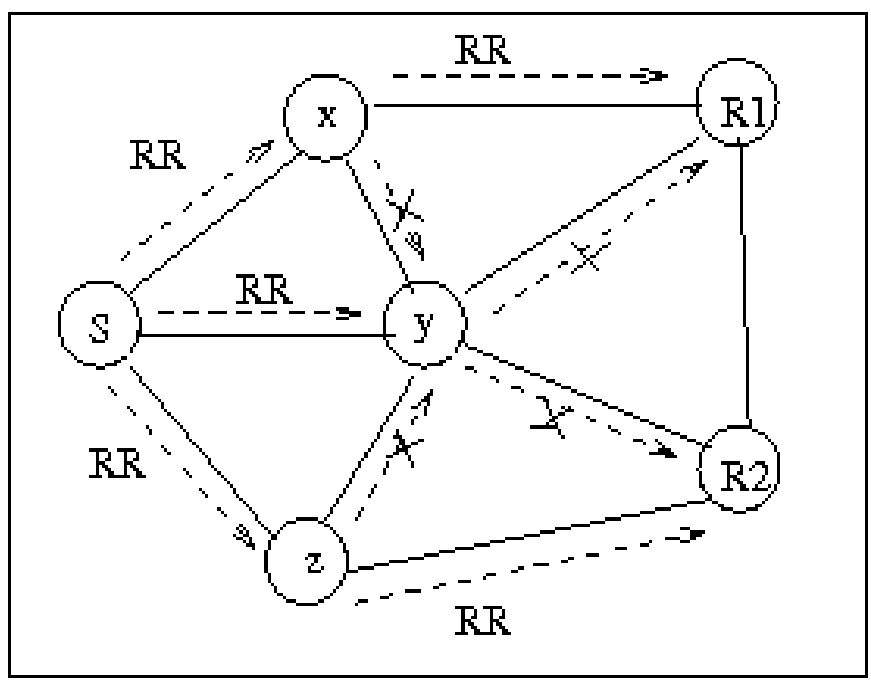

Fig. 2. Route request paths from $\mathrm{S}$ to $\mathrm{R} 1$ and $\mathrm{R} 2$

Fig. 2 illustrates the basic operation of route request phase.

- Source S broadcasts RR packet to discover the route for two multicast receivers $R 1$ and $R 2$.

- Nodes $x, y$ and $z$ receive RR packet from $\mathrm{S}$. These nodes update the paths to $\mathrm{S}$ in its MRIC by using next hop as $S$. Also updates the link stability database and stability factor of next hop in MRIC.

- Node $x$ broadcasts RR packet to $R 1$ and $y$. Node $z$ broadcasts to y and $R 2$. Node y broadcasts to $x, R 2, R 1$ and $z$.

- Node y finds that these packets are duplicates of the same RR packet already received. Thus they will be discarded by node $y$, which is indicated by cross mark in the figure. Similarly nodes $x$ and $z$ discard duplicate RR packets received from y.

- $R 2$ and $R 1$ discards duplicates from nodes $z$ and $x$, respectively.

- $R 2$ and $R 1$ updates MRIC and link stability database.

- Now, $R 2$ and $R 1$ have path to the source $S, R 1-x-S$, $R 1-y-S, R 2-z-S$, and $R 2-y-S$.

2) Reply Phase: Multicast receiver initiates the reply phase. In reply phase, RP packet is generated at a multicast receiver after receiving a RR packet. The following operations are performed in the reply phase. 
1) Receiver prepares RP packet by putting the following information in the packet: its address as receiver address, source address as destination address, makes $\mathrm{RR}=0$, increments sequence number, changes previous node address as next hop address, transmission power and antenna gain. 2) Send RP packet to its neighbors corresponding to group id/source address in MRIC (as prepared during forward movement of RR packets). 3) The node receiving RP packet compares sequence number and next hop address corresponding to group id / destination address (receiver address) with the respective values in MRIC, if available. 4) If sequence number of the arrived RP packet is greater than the sequence number in MRIC, than change the next hop in MRIC as the address from where RP packet has arrived; If the route is not available in MRIC then add the route for group id/ destination address (receiver address) with next hop as the node from where RP packet has arrived. 5) Update the link stability database and stability factor in MRIC. 6) If next hop address in RP packet matches with node address then set FW flag as 01 indicating it as one of the forwarding nodes. 7) Update the previous node address in RP packet to corresponding next hop address for group id/ source address by using MRIC (which is already set during forward movement of RR packet). 8) Send RP packet to its neighbors corresponding to group id/source address in MRIC (as prepared during forward movement of RR packets). 9) Perform steps 3 to 8 until source is reached. 10) If source is not reached within certain hops or could not find any next hop as set by RR packet in MRIC, send RE packet to receiver.

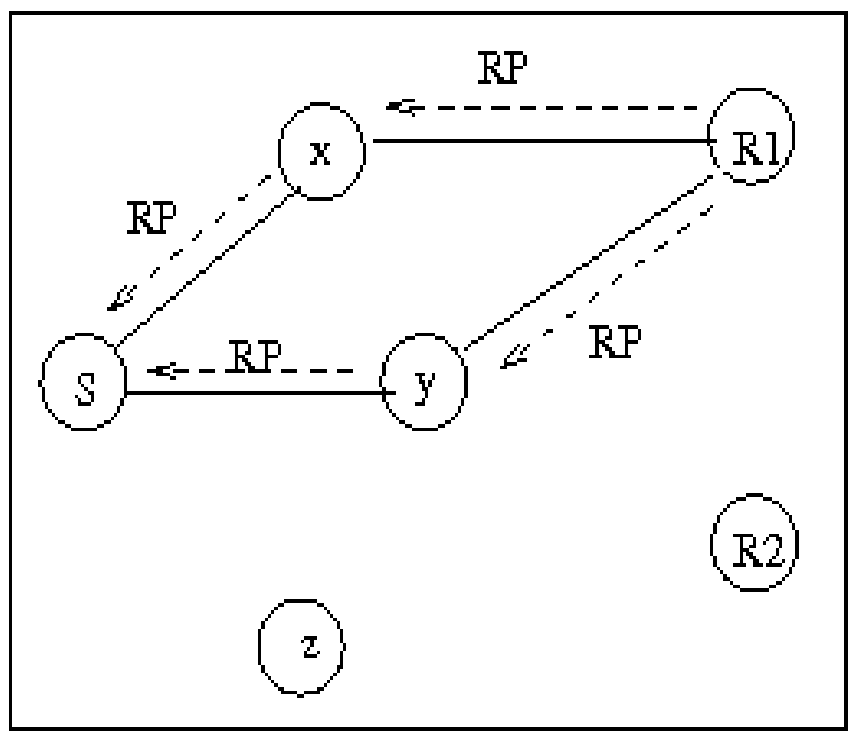

Fig. 3. Reply paths from R1 to S

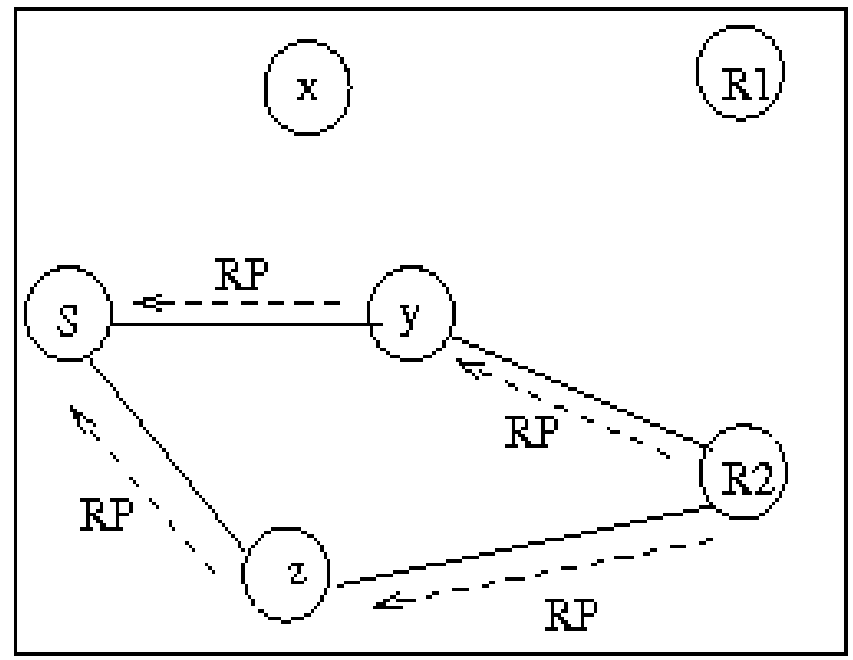

Fig. 4. Reply paths from R2 to S

Let us illustrate the reply phase using Figs. 3 and 4 by using the same example as given in Fig. 2 for request phase. RP packets are sent on the reverse paths from $R 1$ and $R 2$ to mark path from $S$ to receivers and update respective MRIC of the nodes on the path. Reply phase sequence of operation is given as follows.

- $R 1$ broadcasts RP packet to source S through $\mathrm{x}$ and $\mathrm{y}$.

- Nodes $\mathrm{x}$ and y receives RP packet from $R 1$. These nodes update the paths to $R 1$ in its MRIC with next hop as R1.

- Also nodes update the link stability database and stability factor of next hop in MRIC.

- Node x compares next hop address in MRIC with the next hop address of RP packet and if match is found, node $\mathrm{x}$ updates MRIC for $F W$ flag $=01$. This procedure is repeated at node y, and it also sets $F W$ flag $=01$.

- Now, $S$ has paths to $R 1: S-x-R 1, S-y-R 1$.

- Similarly $S$ has paths to $R 2: S-z-R 2$ and $S-y-R 2$.

Finally, the created mesh between source $S$ and multicast receivers $R 1$ and $R 2$ with $\mathrm{x}$ and $\mathrm{y}$ as forwarding nodes is shown in figure 5.

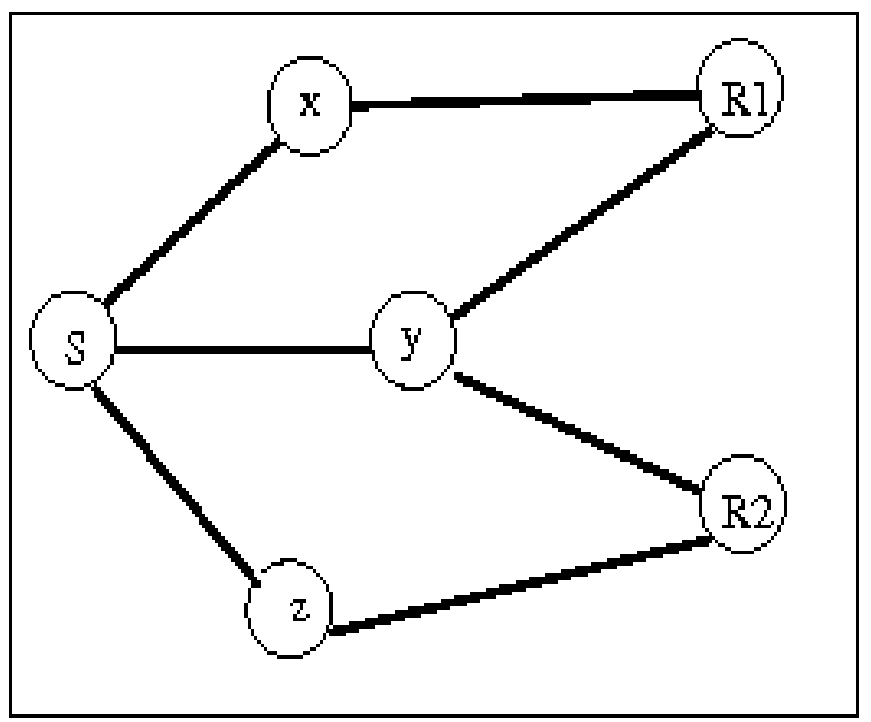

Fig. 5. Mesh created between source and receivers R1 and R2 
In LSMRM, SFN selection among all forwarding nodes in the mesh is an important process since it helps in establishing stable path from receivers to source or vice versa among many alternate paths already found. A forwarding node checks for higher value of stability factor $S_{i j}$ in its MRIC for next hops corresponding to group id / destination address. Forwarding Node selects one of the next hops as SFN using equation 3, and selected SFN FW flag will be set to 10 in its MRIC.

$S F N=\operatorname{maxNEXTHOP}(S)$

For example, in Figure 6, the SFN selected at R1 is node y since it has higher value of $\mathrm{S}=0.6$ than the other node $\mathrm{x}$, whose $S=0.4$. As $y$ belongs to the forwarding node, it updates FW flag = 10 in its MRIC indicating that this node is an SFN.

A complete example of SFN selection from S to R1 and R2 based on stability factor is given in Fig. 7 considering the mesh given in Fig. 5.

\section{F. Mesh Maintenance}

LSMRM detects two types of link failures: 1) link failure between SFN nodes and 2) link failure between a multicast source/receiver and a SFN. In the case of link failure between two SFN's, the failure detecting node will try to find the next stable link in the mesh and route the packet through such a link. In case, if all the forwarding nodes links fail, RE packet is sent to the source to rediscover the routes. The route through the failed link in MRIC will be removed and the FW flag for the chosen next hop will be updated accordingly. When links fail between a SFN node and a multicast node, the multicast node detecting failure deletes the multicast node routing information from its MRIC corresponding to failed SFN. Multicast node updates next hop SFN based on high stability factor. In case, all the forwarding nodes' links connected to multicast node fail, then the node rediscovers the mesh and stable route using RR and RP packets.

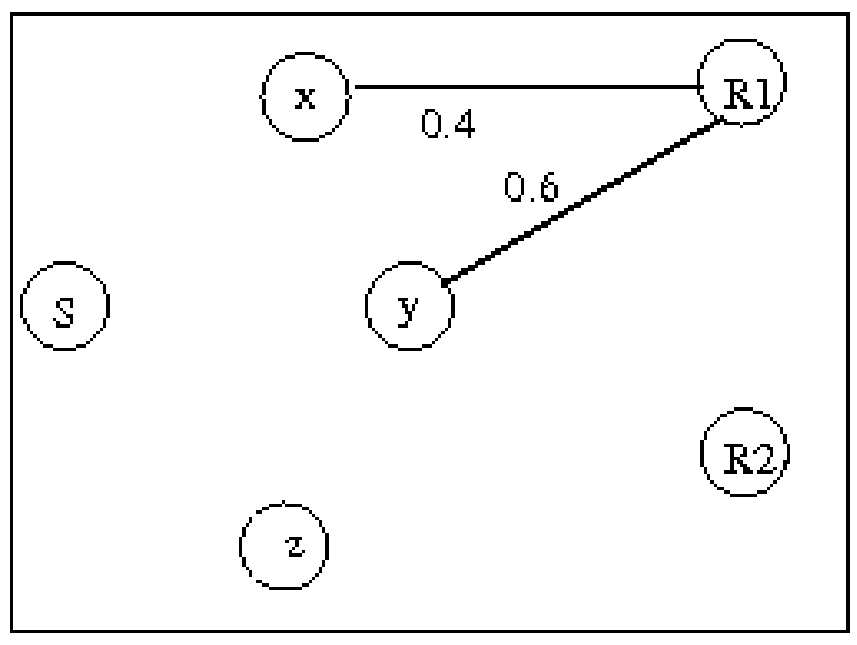

Fig. 6. SFN selection from R1

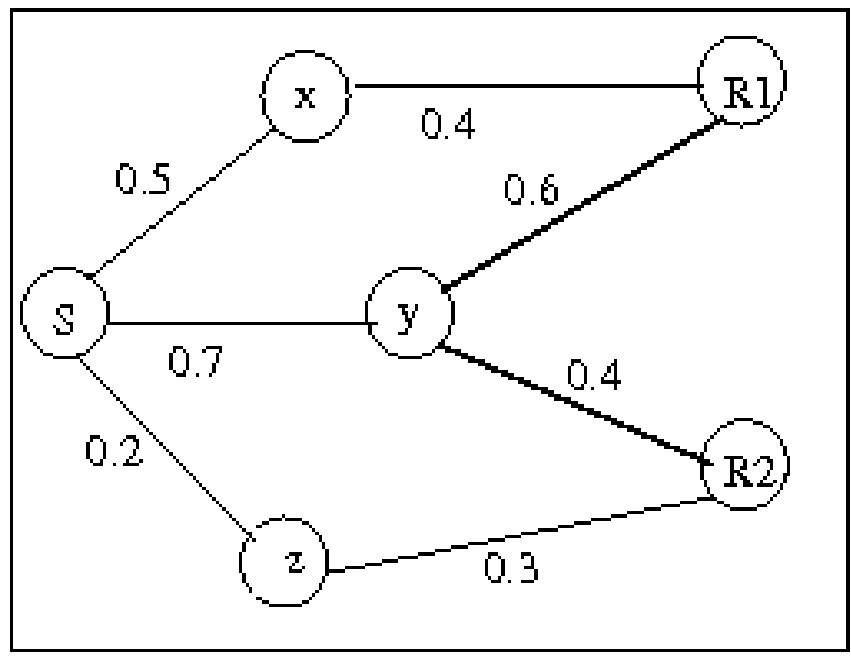

Fig. 7. SFN selection from R2

\section{SIMULATION MODEL}

Proposed LSMRM has been simulated in various network scenarios to assess the performance and effectiveness of the approach. Simulation environment for the proposed work consists of four models: 1) Network model 2) Channel model 3) Mobility model and 4) Traffic model. These models are discussed below.

- Network model: An ad hoc network is generated in an area of $l x b$ square meters. It consists of $n$ number of mobile nodes that are placed randomly within the given area. The coverage area around each node has a bandwidth, $B W_{\text {one }}$-hop that is shared among its neighbors. It is assumed that, the operating range of transmitted power $P_{t}$ for every node is varying and this variation is different for different applications. Other important parameters of the model are the transmitting and receiving antenna gain $G t$ and $G r$ that is set to an appropriate value.

- Channel model: It assumes the free space propagation model defined with following parameters: wavelength of the transmitted signal, system loss L, frequency of transmission $\mathrm{f}$ and distance between the transmitter and receiver $d$. Radio propagation range $r$ for each node changes dynamically, depending upon transmitted power of a node. Each wireless link is associated with a channel noise $N$ that consists of white noise (additive white Gaussian noise, AWGN) and other channel interferences that defines the link quality $q$. To access the channel, ad hoc nodes use CSMA/CA MAC layer protocol to avoid possible collisions and subsequent packet drops. Received signal strength is measured in terms of signal-to- noise ratio $(S N R)$. A packet will be accepted if it is received with $S N R$ higher than the fixed receiver threshold $R x T h$. Packet propagation delays (per hop) are generated proportional to distance between the nodes, i.e., we consider $m$ sec. per meter.

- Mobility model: We use a random way-point (RWP) mobility model based upon three parameters; speed of movement, direction for mobility and time for mobility. In RWP, Each node picks a random destination uniformly within an underlying physical space, and travels with a speed $v$ whose value is uniformly chosen in 
the interval 0 to $V \max$. Vmax is some parameter that can be set to reflect the degree of mobility. Upon reaching the destination, the node pauses for a time period $Z$, and the process repeats itself afterwards. Eight directions are considered for movement of nodes: east, west, north, south, north-east, north-west, south-east and south-west. A node starts with a random direction to pick its neighbor in that direction and this process continues till it reaches the boundary and after reaching the boundary it bounces back.

- Traffic model: Traffic model used is constant bit rate model that transmits a certain number of fixed size packets, Trpkts. Various multicast group sizes GpSize are defined to assess the performance of packet delivery ratio, control overhead and packet delay with group sizes. The packets are transmitted with bandwidth $b t$ bps.

\section{A. Simulation Parameters}

The following parameters are used for simulation. $l=1000$ meters, $b=1000$ meters, $n=50$ to 250 with an increment steps of $50, B W_{\text {one-hop }}=50 \mathrm{Mbps}, P_{t}=5 \mathrm{~W}$ to $50 \mathrm{~W}$ with an increment step of 5W. $G_{t}=G_{r}=1$. $\lambda=0.100 \mathrm{~m}$ to $0.135 \mathrm{~m}$, $L=1, f=200 \mathrm{MHz}, d=20 \mathrm{~m}$ to $500 \mathrm{~m}, r=200 \mathrm{~m}$ to $600 \mathrm{~m}, q=$ 0.2 to 0.6. Receiver Threshold $R x T h=0.05 \mathrm{~W}$., $m=0.001$ sec., Speed of a node $v=[0, V \max ]=[0 \mathrm{~m} / \mathrm{s}, 25 \mathrm{~m} / \mathrm{s}]$ and $Z=$ $0.2 \mathrm{~ms}, T r p k t s=1000$, GpSize $=5$ to 50 and $b t=2 \mathrm{mbps}$.

\section{B. Performance Measures}

The following performance measures are considered in simulation since our objective was to increase the packet delivery ratio (PDR) and reduce control overheads and packet delay. Increased PDR and reduced packet delay improves network throughput and reduced control overheads reduce bandwidth overheads for route discovery and maintenance.

Packet delivery ratio (PDR): It is defined as the sum of number of packets received at all the multicast receivers to the product of number of packets sent at source and number of multicast receivers.

Control overhead: It is the total number of control packets needed to establish a stable route from source to the multicast receivers.

Packet delay: It is defined as the average time taken to transmit predefined number of packets from source to multicast destinations for various group sizes.

\section{RESULTS}

In this section, we discuss the results obtained with proposed scheme LSMRM and ODMRP. Three categories of results are analysed: 1) Analysis of PDR, 2) Analysis of control overhead and 3) Analysis of packet delays.

\section{A. Analysis of PDR}

Three cases of PDR analysis are performed: 1) Effect of transmitting power at a node on PDR for varying number of nodes of certain group size, 2) Effect of multicast group size on PDR for varying number of nodes, and 3) Effect of group size over PDR for varying speed of nodes for a fixed group size. Figs. 8 to 13 depict the analysis of PDR.

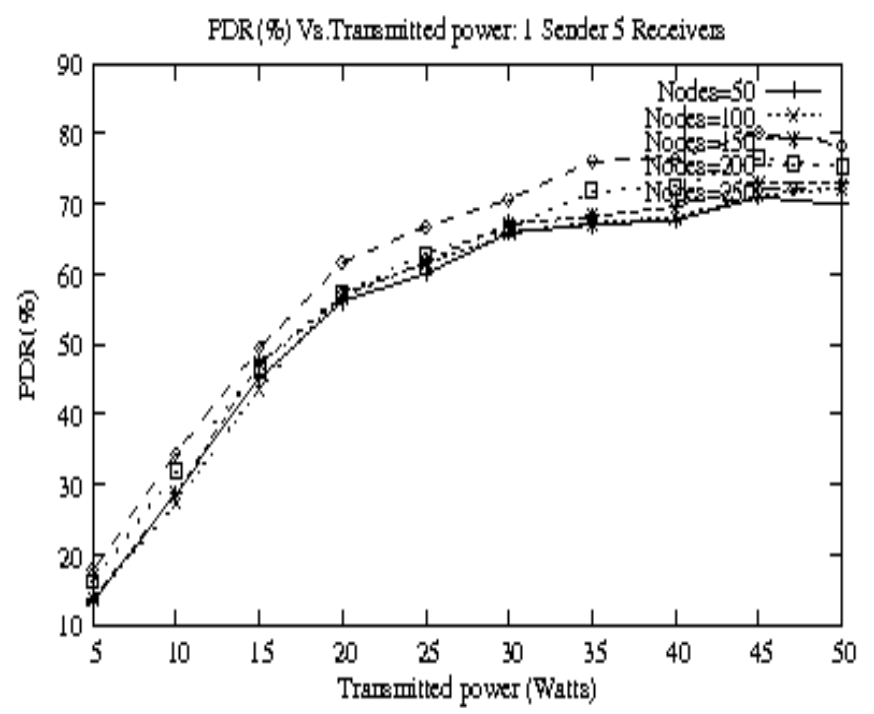

Fig. 8. PDR Vs. Transmitted power

Fig. 8 shows that there is a increase in PDR as power at the transmitting node increases (for 1 source, 5 receivers for transmitting 1000 packets). We also observe that, as the number of nodes increase (from 50 to 250) in the network, the

delivery of packets increases. As number of nodes increase, there is a possibility of decreased hop length between the nodes, which enhances the link stability and reduces packet drops. The noise signal dominates at low transmission power levels than at higher levels that resulted in less PDR at lower power levels. The increase in PDR is fairly stable at higher power levels (more than 20W), where the network reaches saturation level since the traffic exceeds $B W_{\text {one-hop }}$ at every node leading to constant packet drops.

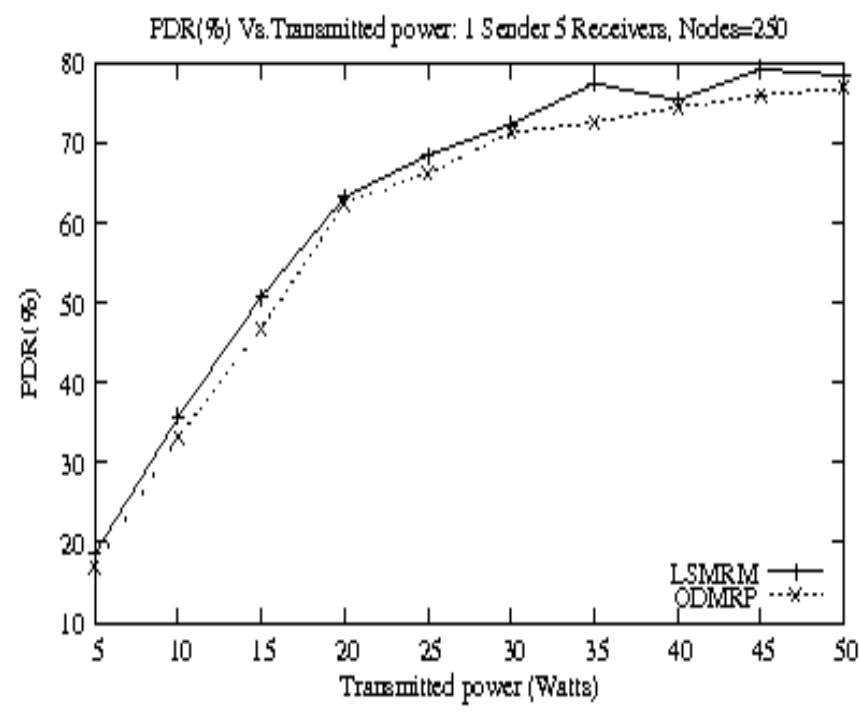

Fig. 9. PDR Vs. Transmitted power (nodes=250)

We also compared the PDR performance of LSMRM with DMRP for 250 node network with 1 source and 5 receivers and 5000 packets transmission (see figure 9). It is observed that LSMRM gives better PDR compared to ODMRP since ODMRP does not consider link stability for data transmission i.e., ODMRP treats all links in the mesh in the same manner. 


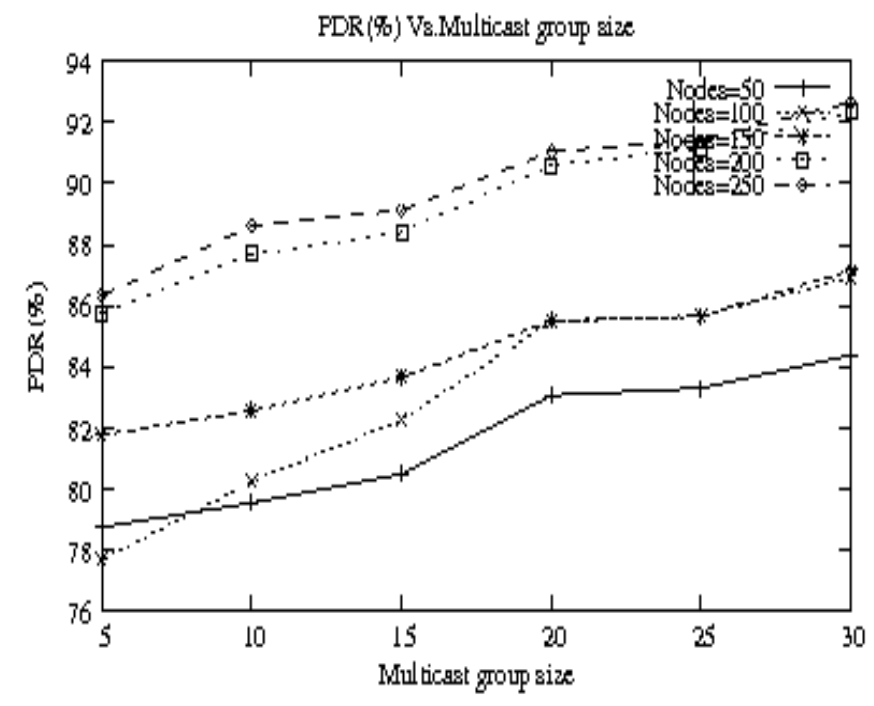

Fig. 10. PDR Vs. Group size

PDR increases as the group size increases (see figure 10) with each group member source transmitting the packets. This is because the number of forwarding nodes will increase with increase in group size; similarly probability of having stable forwarding node will be more. Also, it is observed that PDR is increased with increase in number of nodes in the network due to more stable links coming in the mesh and high probability of connectivity.

LSMRM provides better PDR compared to ODMRP as shown in figure 11, in which the results are given for 200 node network. For the group size of 5, PDR is 86 percent for LSMRM whereas it is 74 percent for ODMRP. This is because, ODMRP relies on links which are vulnerable to packet drops in contrast to LSMRM that are established based on high stability factor. The gap between PDR curve of LSMRM and ODMRP decreases with increase in group size since connectivity improves in ODMRP with increase in group size. But LSMRM maintains consistent PDR with increase in group size.

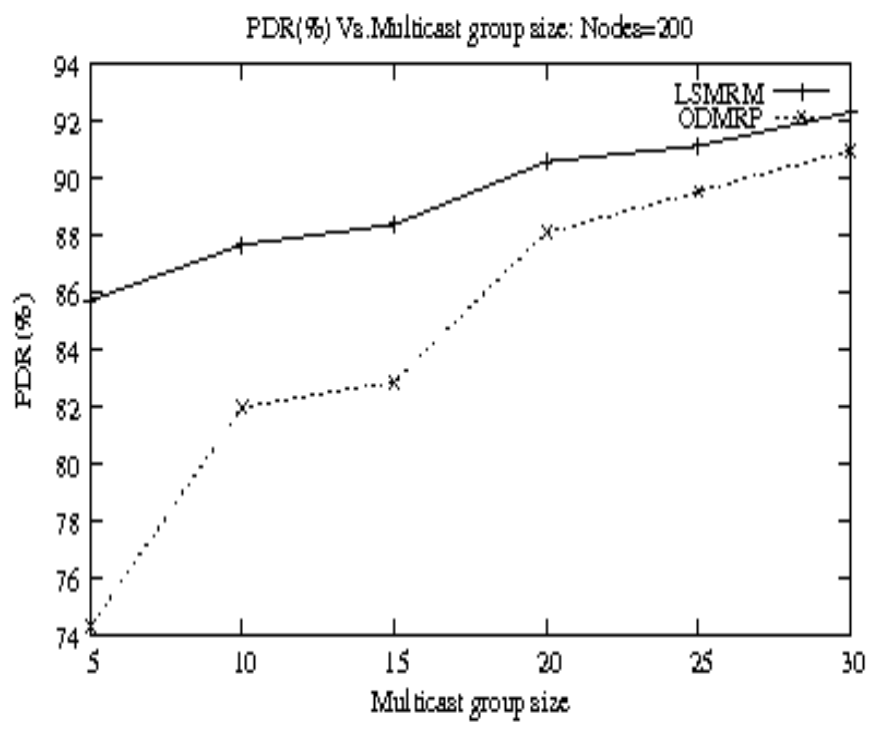

Fig. 11. PDR Vs. Multicast group size (nodes=200)

Fig. 12 depicts the effect of mobility on PDR for different group sizes. Lower mobility of nodes corresponds to higher PDR. Group size increase does not cause perfect increase in
PDR, but PDR oscillates with increase in group size and the mobility. Higher mobility causes slightly higher oscillations than lower mobility. Mobility of node triggers new SFN selection to find a new stable path towards the source causing the packet drops and hence decrease in PDR. PDR of SMRM compared to that of ODMRP is better with node mobility since ODMRP uses route refresh cycles to handle mobility of nodes (see figure 13). After a group size of 25, the PDR falls to lower value since forwarding node may select less stable link due to false power level measurement at a moving node interface. LSMRM provides around 15 percent increase in PDR compared to ODMRP.

\section{B. Analysis of Control Overhead}

Fig. 14 shows that number of control packets for mesh establishment increases with the increase in number of nodes in the network. For given number of nodes, control packets increase with increase in group size. It is to be noticed that, when network size increases (beyond 200 nodes), the number of control packets decrease at higher multicast group sizes. his is due to the possibility of selecting the same SFN by two or more receivers since this SFN happens to be the nearest neighbor for all those receivers as per the SFN selection rule and thus reducing the number of control packets to construct a mesh. LSMRM uses less number of control packets compared to the overheads required by ODMRP for a group size of 5 as shown in Fig. 15.

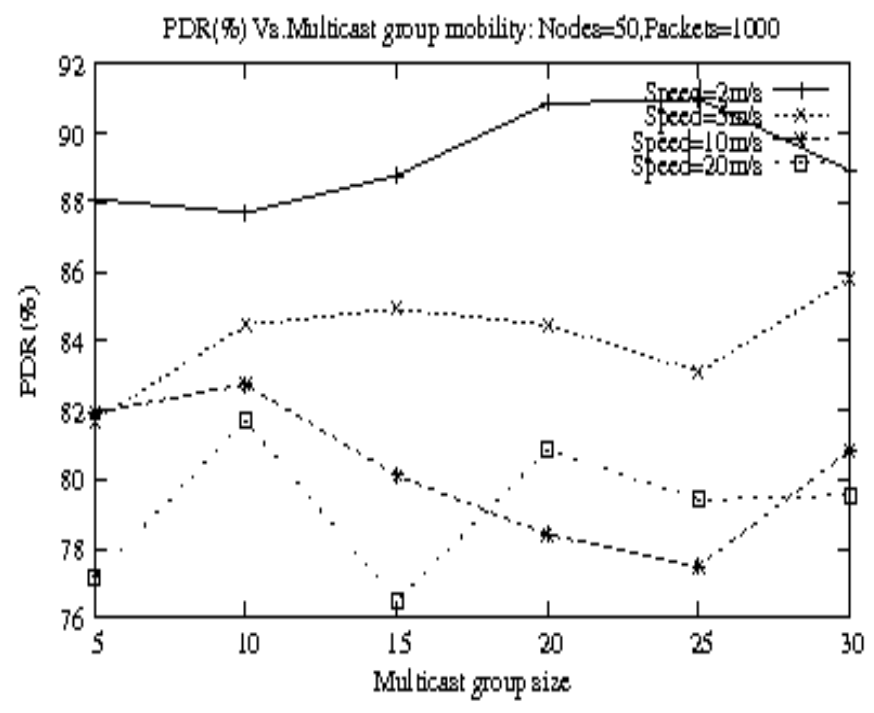

Fig. 12. PDR Vs. Multicast group size

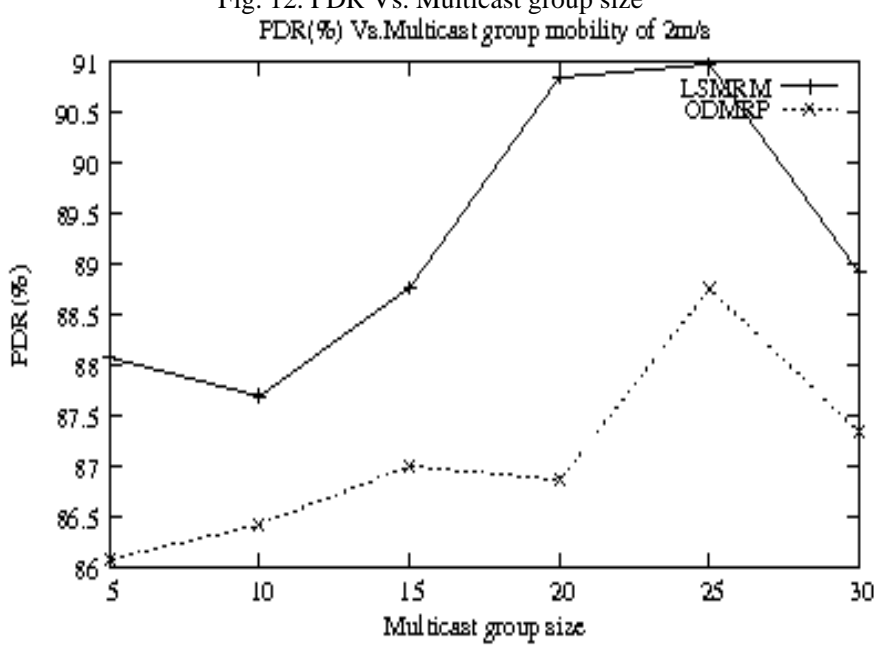


Fig. 13. PDR Vs.Multicast group size (comparison with ODMRP; mobility= $2 \mathrm{~m} / \mathrm{s})$

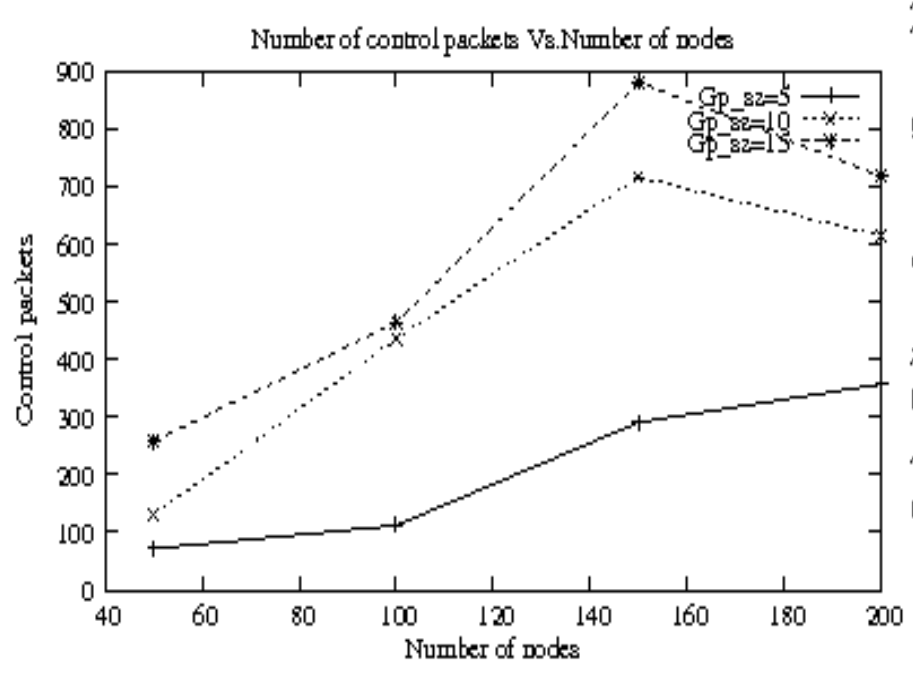

Fig. 14. Control Packets Vs. Number of nodes

\section{Analysis of Packet Delay}

Fig. 16 shows the packet delays to transmit 1000 packets for varying mobility of nodes. For group sizes of 5 and 10, the time required to transmit the packets to all destinations in LSMRM is less than that of ODMRP because LSMRM finds nearest stable link to forward packets when nodes start moving, where as in ODMRP, node mobility triggers new path set up.

During high node mobility (more than $15 \mathrm{~m} / \mathrm{s}$ ), link failures will cause packet delays to increase. In LSMRM, the SFN detecting link failure will try to find the next stable link in the mesh and route the packet through such a link. In case, if the entire forwarding node's links fail, RE packet is sent to the source to rediscover the routes. In such a condition, the packet delay of LSMRM almost reaches nearer to the packet delay of ODMRP.

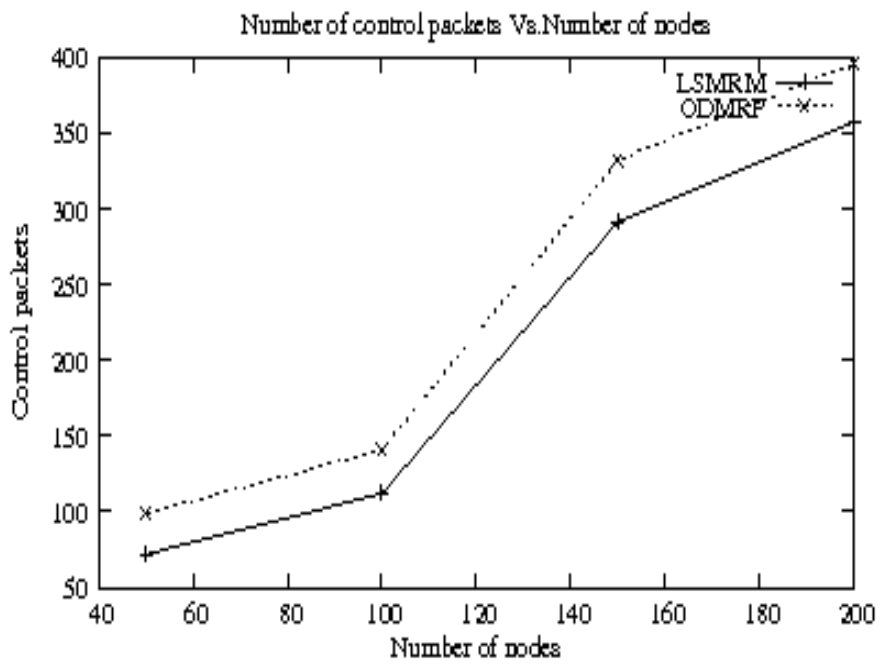

Fig. 15. Control packets Vs. Number of nodes (comparison with ODMRP; group size=5)

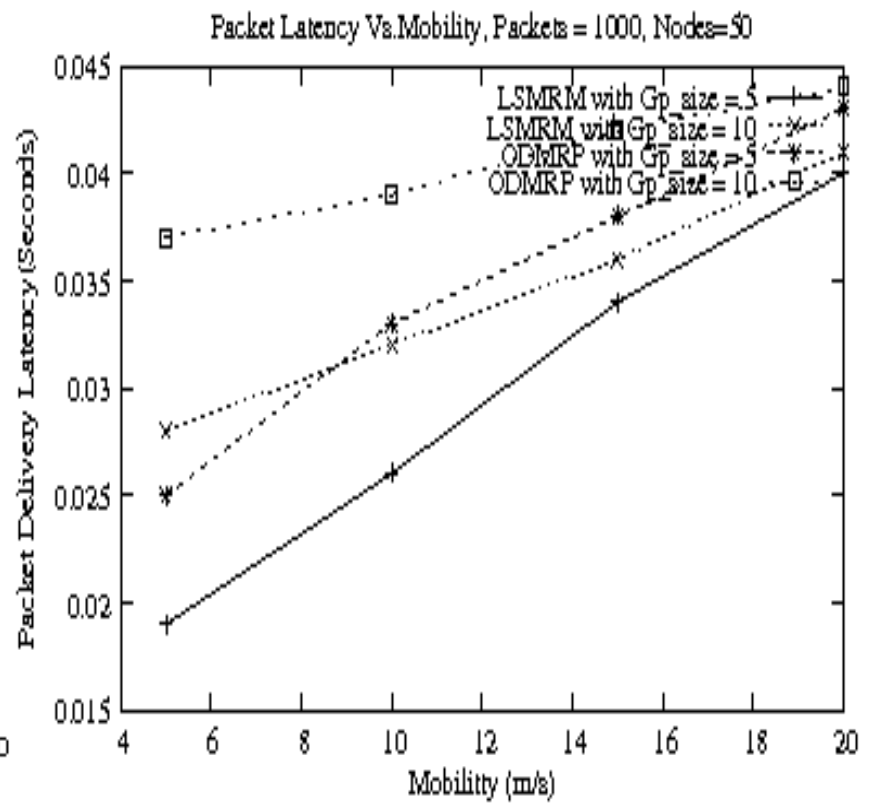

Fig. 16. Packet delay Vs. Mobility (comparison with ODMRP; group size $=5$ AND 10)

\section{CONCLUSIONS}

In this paper, we proposed stability based multicast routing scheme in MANET. It finds the multicast routes to receivers by using route request and route reply packets with the help of routing information maintained in MRIC and link stability parameters maintained in link stability database on every node in a MANET. Multicast mesh of alternate paths between every source-destination pair is established in mesh creation phase. Stable path within a mesh is established by choosing an SFN that possess higher value of link stability among its neighbors. This assures better quality of links and minimizes the possibility of link failures and the overhead needed to construct the paths. Link failure conditions are notified to the source with route error packets so as to enable the source to start route discovery for new route establishments. Extensive simulation is performed to assess the network with three performance metrics such as packet delivery ratio, control overhead and packet delay and the comparison is made with ODMRP. The proposed scheme showed significant improvements in terms of packet delivery ratio, control overheads and packet delay as compared to ODMRP. We would like extend the work by employing cognitive agents to perform mesh creation and stable route selection by embedding intelligence into the agents, which can improve the scalability, flexibility (bandwidth constrained routing, delay constrained routing, cost constrained routing) and customization services for multicasting.

\section{REFERENCES}

[1] Kaan Bur, Cem Erosy, "Ad Hoc quality of service multicast routings", Computer communications, Vol. 29, 2005, pp. 136-148.

[2] Hui Cheng a, Jiannong Cao, Xingwei Wang, "A fast and efficient multicast algorithm for QoS group communications in heterogeneous network", Computer communications, Elsevier, Vol. 30, 2007 pp. 2225-2235.

[3] Khalid A. Farhan, "Network sender multicast routing protocol", Proceedings of seventh IEEE International conference on networking, 2008, pp. 60-65. 
[4] Harleen Dhillon, Hung Q. Ngo, "CQMP: A mesh-based multicast routing protocol with consolidated query packets", IEEE Communications Society /WCNC 2005, pp. 2168-2174.

[5] Luo Junhai, Ye Danxia, Xue Liu, and Fan Mingyu "A Survey of Multicast Routing Protocols for Mobile Ad-Hoc Networks", IEEE Computer Communications Surveys and Tutorials Vol. 11, No. 1, First Quarter 2009, pp. 78-91.

[6] Jason Xie et al, "AMRoute: Ad hoc multicast routing protocol, mobile networks and applications”, Kluwer Academic Publishers, 2002, pp. 429-439.

[7] Wu, C.W and Tay, Y.C., ”AMRIS: A multicast protocol for ad hoc wireless networks", IEEE Proceedings of Conference on Military Communications, MILCOM 1999, Vol.1, pp. 25-29.

[8] Elizabeth M. Royer and Charles E. Perkins, "Multicast operation of the ad-hoc on-demand distance vector routing protocol", Mobile Computing and Networking, 1999, pp. 207-218.

[9] William Su Sung-Ju Lee, Mario Gerla, "On-demand multicast routing protocol in multihop wireless mobile networks", Mobile Networks and Applications, Kluwer Academic Publishers, Vol. 7, 2002, pp. 441-453.

[10] Ching-Chuan Chiang, Mario Gerla, Lixia Zhang, "Forwarding group multicast protocol (FGMP) for multihop, mobile wireless networks", Cluster Computing, Vol. 1, No. 2, 1998, pp. 187-196.

[11] Ewerton L. Madruga, J. J. Garcia-Luna-Aceves, ”Scalable multicasting: the core-assisted mesh protocol", Mobile Networks and Applications, Vol. 6, No. 2, 2001, pp. 151-165.

[12] Seungjoon Lee, Chongkwon Kim, "Neighbor supporting ad hoc multicast routing protocol", Proceedings of the 1st ACM international symposium on Mobile ad hoc networking and computing, IEEE Press, 2000, pp. 37-44.

[13] Young-Bae Ko, Nitin H. Vaidya, "Geocasting in mobile ad hoc networks: Location-based multicast algorithms", Proceedings of the Second IEEE Workshop on Mobile Computer Systems and Applications. 1999, pp. 101-107.

[14] B. S. Manoj Subir Kumar Das, C. Siva Ram Murthy, "A dynamic core based multicast routing protocol for ad hoc wireless networks", Proceedings of the 3rd ACM International Symposium on Mobile Ad Hoc Networking and Computing, Switzerland. 2002, pp. 24-35.

[15] Riaz Inayat, Usman Haider Gardezi, Ahmad Raza Shahid, "Adaptivemesh based routing for efficient multicasting in highly mobile ad hoc networks", Proceedings of 2nd International Conference on Emerging Technologies, IEEE-ICET 2006, pp. 493-500.

[16] E. Baburaj, V. Vasudevan, "An intelligent mesh based multicast routing algorithm for MANETs using particle swarm optimization", International Journal of Computer Science and Network Security, (IJCSNS), Vol.8, No.5, 2008, pp. 214-218.

[17] Moustafa H, Laboid, H, "A multicast on-demand mesh-based routing protocol in multihop mobile wireless networks", Proceedings of IEEE 58th Vehicular Technology Conference, VTC 2003, Vol.4, No.6, 2003, pp. 2192-2196.

[18] Yao Zhao, Leiming Xu, Meilin Shi, ”On-demand multicast routing protocol with multipoint relay (ODMRP-MPR) in mobile ad-hoc network”, Proceedings of ICCT 2003.

[19] MohammadReza EffatParvar, Naser Yazdani, Farshad Lahooti, Mehdi EffatParvar, "Link Stability Approach and Scalability Method on ODMRP in Ad Hoc Networks" Seventh Annual Communication Networks and Services Research Conference, CNSRC 2009, pp.416-421.

[20] Hao Xu, Dejun Mu, ”A Cluster Based Stable Multicast Routing Protocol in Ad Hoc Network", IEEE/IFIP International Conference on Embedded and Ubiquitous Computing, EUC 2008, Vol. 2, pp.723-728.

[21] Rajashekhar Biradar, Sunilkumar Manvi, Myalara Reddy, ”Mesh based multicast routing protocol in mobile ad hoc networks", Proceedings of National Conference on Computer Networks, NCCN 2009, Bangalore, pp.17-22.

[22] I. Stepanov, D. Herrscher, K. Rothermel, "On the impact of radio propagation models on MANET simulation results", Proceedings of 7th International Conference on Mobile and Wireless Communications Networks (MWCN 2005), Marrakech, Morocco, September 2005.

[23] Qing Dai, Jie Wu, "Computation of minimal uniform transmission range in ad hoc wireless networks", Cluster Computing, Springer Science, Vol.8, 2005, pp.127-133.

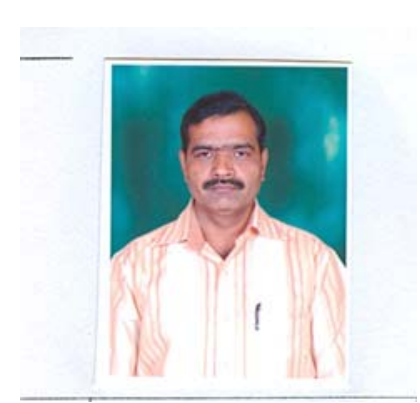

Rajashekhar C. Biradar has completed his B.E (ECE) and M.E (Digital Electronics) from Karnataka University, Dharwad, India. He is a research scholar at Visvesvaraya Technological University (VTU), Belgaum, India. Currently he is working as faculty in Reva Institute of Technology, Bangalore, India. He has some National/International Journal and Conference publications to his credit. His areas of interest include group communication, multicast routing in MANET, wireless Internet, software agents etc. He is a Member IETE (India), Member IE (India) and Member ISTE (India)

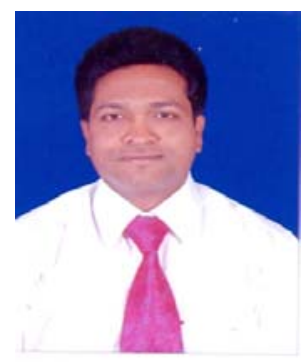

Sunilkumar S. Manvi has obtained his $\mathrm{PhD}$ from Indian Institute of Science (IISc), Bangalore in 2004. To his credit, he has authored 3 books, about 30 National/International Journal publications and about 85 National/International Conference publications. He is guiding $7 \mathrm{PhD}$ students. Currently he is working as Professor and Head, Department of ECE, Reva Institute of Technology and Management, Bangalore, India. His areas of interest include agent technology, grid computing, vehicular ad hoc networks, wireless sensor and ad hoc networks, e-commerce and U-commerce. etc. he is member of IEEE (USA), Fellow IETE (India), Fellow IE(India) and Member ISTE (India).

Mylara Reddy has completed his M. Tech (Computer Networks) from Visvesvaraya Technological University (VTU), Belgaum, India. Currently he is working as faculty in Department of Information Science and Engineering, Reva Institute of Technology and Management, Bangalore, India. His research interests include wireless mesh networks, agent technology, ad hoc networks, etc. 